\title{
Some aspects of buoyancy adaptations of chaetognaths
}

\author{
Helga Kapp* \\ Biologische Anstalt Helgoland; Notkestraße 31, D-W-2000 Hamburg 52, \\ Federal Republic of Germany \\ and \\ Zoologisches Institut und Zoologisches Museum der Universität Hamburg; \\ Martin-Luther-King-Platz 3, D-W-2000 Hamburg 13, Federal Republic of Germany**
}

\begin{abstract}
Chaetognaths mainly keep to a preferred depth range by movement and resistance achieved by body shape. Some species, for example Sagitta lyra and $S$. hexaptera, developed conspicuous, voluminous, gelatinous inner parts in the lateral fins. These are considered an aid toward buoyancy, as they compensate the increasing density caused by maturing gonads. Probably, the small size of the sexual organs of $S$. enflata can also be regarded as a buoyancy adaptation.
\end{abstract}

Most chaetognath species live in the pelagic layers of the oceans. Noteworthy is the fact that all chaetognaths are rather similar, that is, their basic structural pattern is only slightly modified, which is perhaps a reflection of the minor environmental differences within the oceans. Apart from this external uniformity, chaetognaths have developed a number of slight differences and special structures of organs. I am going to deal with a few of these structures, especially the gelatinous masses in the lateral fins and the small size of the sexual organs, which I consider to be species-specific buoyancy adaptations.

Plankton organisms need to keep to the depth range in which they flourish. As the density of the surrounding milieu is determined by temperature, salinity and pressure, plankton organisms attain their ability to stay in a preferred depth by movement, resistance due to body shape, incorporation of substances of low specific gravity (gases, lipids, ions, jelly), or a combination of these different techniques. In addition to their physical functions, these buoyancy adaptations are comprehensible when we consider their evolutionary development.

Chaetognaths are considered to be very well adapted to their planktonic, carnivorous way of life. These small tubes, full of fluid, with compact head, slowly sink down in the water in an oblique or horizontal position (negative buoyancy) and ascend with fast movements which appear, to the naked eye, to be vibrations. Many a species is able to "stand" or to "lie", motionlessly to our eyes, in any position, even perpendicularly, in the water. In addition, they are all able to dart forward very rapidly in any direction in order to escape predators or to seize prey (vernacular name "arrow worms"). Their fins have mechanical functions for locomotion and are constructed like "Tragflächen" (bearing

\footnotetext{
- Member of the Taxonomy Group at the Biologische Anstalt Helgoland

- Address for correspondence 
surfaces): streamlined, without musculature, stiffened by two layers of fin rays, and with a wedge-shaped, gelatinous attachment to the body. Thus chaetognaths keep to their preferred depth range by movement and resistance achieved by body shape, and many a species also by more or less well developed buoyancy devices.

Probably, the body fluid in many species has a density similar to that of seawater, but a series of species develops large vacuolated cells in the side walls of the gut - sometimes these cells nearly fill the whole body cavity in the trunk. The fluid in the vacuolated cells contributes to reduce the specific gravity. In Sagitta elegans, the body fluid was found to be less dense than seawater, because $\mathrm{NH}^{+}$replaces $\mathrm{Na}^{+}$. Eukrohnia hamata, for example, contains oil globules in the gut, probably developed more as an energy reservoir than as an aid to floating. Also, the importance of the alveolar tissue, a character of many chaetognath species, is uncertain. To my knowledge, its specific structure and function are not yet examined on species level. The alveolar tissue is situated at the neck, trunk or tail; it runs laterally along the trunk or the whole body or it is a voluminous cover surrounding the epipelagic species Pterosagitta draco as well as the bathypelagic species Heterokrohnia involucrum. The alveolar tissue of the epipelagic $P$. draco could be regarded as an aid to buoyancy, whereas the thin cover of alveolar tissue enwrapping juvenile Eukrohnia specimens within the breeding sacks could be considered as a mechanical protection. More or less voluminous gelatinous masses are produced in the lateral fins of some Sagitta species with increasing maturity, for instance in $S$. lyra, $S$. gazellae, S. hexaptera, S. enflata, S. planctonis, and S. zetesios; in the two latter species the thick gelatinous fins are combined with an extended well developed alveolar tissue and vacuolized gut cells. Probably, the development of jelly from the gelatinous matrix of the lateral fins is a method to reduce the specific gravity, thus countering the increasing weight of gonads during maturation. Other groups of plankton organisms also use jelly as a buoyancy technique (medusae, salps, ctenophores), since the density of jelly is lower than that of sea water.

During gonad development, the specific gravity of the chaetognaths increases. Living substance, consisting mainly of protein, has a higher density than oceanic sea water. The tissues of testes, seminal vesicles, sperm and ovaries, seminal receptacles, crescents, and ova cause an increase in density of the animals, because the lighter body fluid is partly substituted by heavier solid tissues. In experiments, completely mature ovaries and tails totally filled with clusters of developing sperms proved to be of distinctly higher density than the remaining chaetognath bodies.

As fresh specimens of $S$. hexaptera, $S$. enflata, and $S$. lyra were not at my disposal, I tested $S$. setosa on Helgoland. The female gonads and the tails filled with clusters of spermatocytes and sperm floated in sea water solution of about $85-90 \%$ salinity $\left(20^{\circ} \mathrm{C}\right)$, the remaining bodies floated in water of about 55-60\% salinity.

It is unknown, whether there is any correlation between the species-specific length of tail, the quantity of male gametes and the ability to counteract the increase in weight, respectively in density.

The large variations in female gonad size within different species is conspicuous; the ovaries can reach the neck region or only occupy the posterior part of the trunk; consequently, the density increase during maturation is different for different species. Species adapted to a restricted depth range need to balance out this increasing density. Species with strong longitudinal musculature can prevent sinking to intolerable depths 
by active movement; species with poorly developed musculature have to compensate the increasing specific gravity by additional techniques.

S. lyra, S. enflata, and S. hexaptera (Fig. 1), which are found in the tropicalsubtropical zones of all oceans, belong to these last mentioned species with a weak longitudinal musculature, a voluminous body stiffened by turgescence, and a tail segment of only $15-20 \%$ of the whole body length.

The occurrence of $S$. lyra is restricted to the epi- and mesopelagic layers. Its long lateral fins with a rayless proximal part and a rayless connecting bridge between anterior
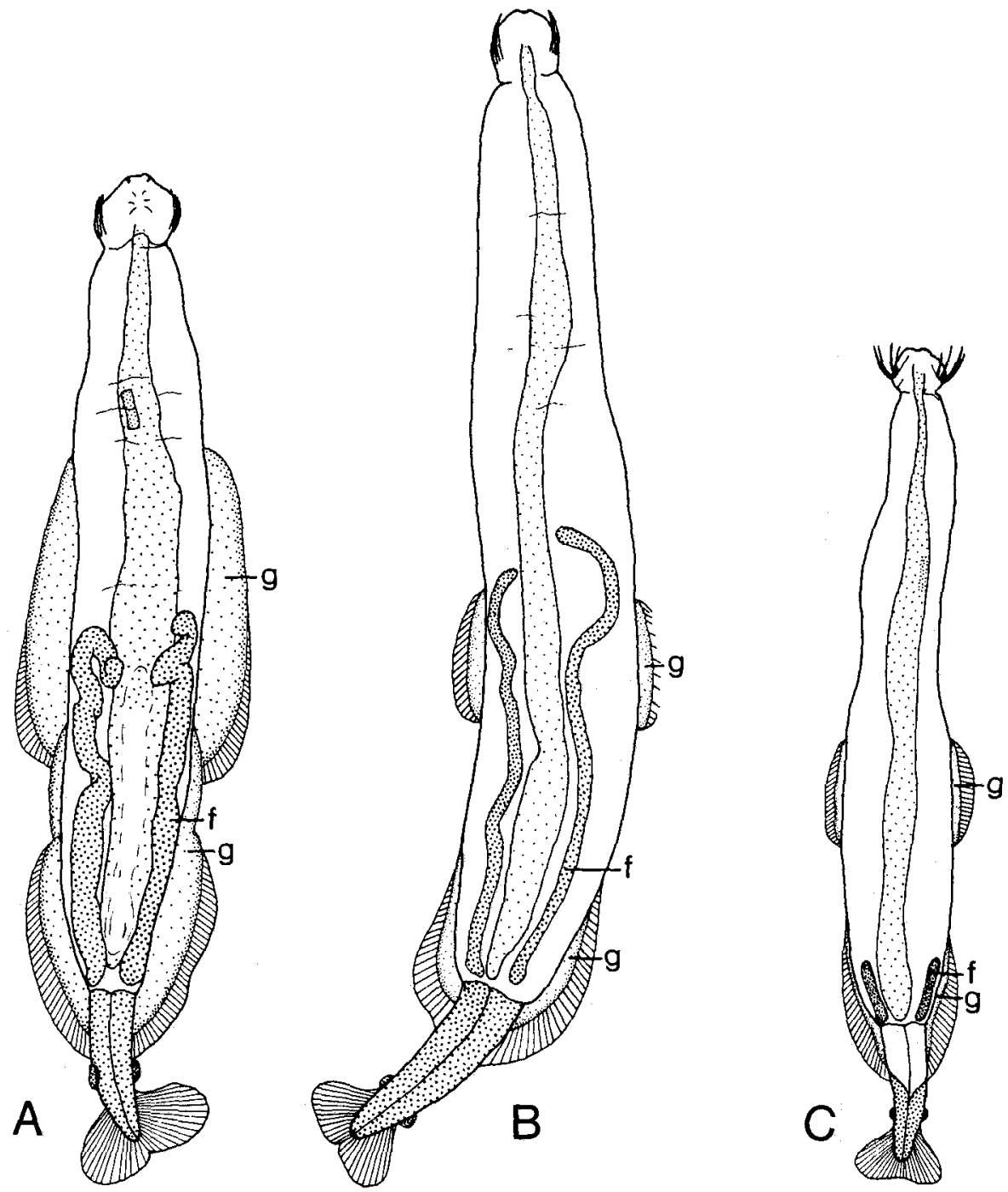

Fig. 1. A: Sagitta lyra $(26 \mathrm{~mm})$. B: S. hexaptera $(32 \mathrm{~mm})$. C: $S$. enflata $(22 \mathrm{~mm}) . \mathrm{g}=$ gelatinous substance, $f=$ female gonad 
and posterior fins are an important character of $S$. lyra. The expanded gelatinous inner parts of the fins are conspicuous in mature specimens, so are the female sexual organs that almost reach the ventral ganglion, thereby nearly occupying the whole space between gut and body wall, thus causing an essential increase in density.

The following hypothesis is here advanced: the gelatinous substance of the fins developes from the wedge of gelatinous matrix and compensates the increase of specific gravity during gonad development. This gelatinous matter enables the members of this species to stay in the warm-water sphere during their entire life cycle. S. gazellae from Antarctic waters must be mentioned here as an example that demonstrates the contrary. Although $S$. gazellae is of larger size (up to $120 \mathrm{~mm}$ ) than $S$. lyra (up to $45 \mathrm{~mm}$ ), its body constitution is very similar. The relative jelly volume of the lateral fins is distinctly lower in $S$. gazellae. During progressing maturity, S. gazellae sinks down into deeper and deeper waters. This species does not need to keep to a certain level, because environmental factors such as temperature and salinity differ only slightly - even for marine conditions - between surface and bottom in Antarctic-Subantarctic waters.

$S$. enflata is adapted to the uppermost layers of the warm-water sphere which has the lowest density and a vertical range of only $100 \mathrm{~m}$ to $200 \mathrm{~m}$ at most. S. enflata does not even tolerate the environmental conditions of the mesopelagic layer with its lower temperatures. It has no significant buoyancy jellies, but only narrow and thin gelatinous inner parts in its very short anterior and posterior fins. The quantity of gelatine which could be produced in such short fins would probably be too small to compensate the weight of heavy gonads. However, one character of the structural pattern of $S$. enflata is unique among all the chaetognath species: developing spermatoids do not float in the whole cavity of the tail segment as in the other arrow-worms, but are restricted to a limited space in the posterior half of the tail segment. The female gonads are small as well. This reduction of sexual products can be understood as a restriction of weight not burdening the animals any more than they can compensate by active movements. Biotechnically this is quite a different method to prevent sinking than the compensation of increasing specific gravity by incorporation of low-density substances.

$S$. hexaptera is only observed in the epi- and mesopelagic layers, but not in greater depths. Its structural pattern can be understood as a construction combining both of the above described "buoyancy techniques". S. hexaptera has short fins, but at maturity these fins contain a conspicuous quantity of jelly, though not as voluminous as in S. lyra. The female organs nearly reach the level of the ventral ganglion as in $S$. lyra, but as narrow cords they are far from filling out the whole trunk cavity. They seem to be reduced in volume and, accordingly, in weight, thus causing only a slight increase in specific gravity. I suppose that this slight increase in density can be compensated by the gelatinous substance in the fins. Due to the shortness of the fins, the incorporation of light material would not achieve enough lift to balance the weight of long, thick, heavy gonads.

I am aware that the buoyancy adaptations of three Sagitta species are only a tiny sector of a great circle of questions. Further investigations are needed to gain more exact knowledge on the density of the various kinds of tissue, including ova, the density and chemical composition of the body fluid, the relations of body proportions and density, the species-specific structure of the alveolar tissue, the mode of movement, and the mode of life of many chaetognath species. Not only the structure, but also the function of the 
different features should be studied in order to understand in which way the different factors of the environment and of the interior of plankton organisms are balanced to a viable poise and in which way the species diversity developed in the marine pelagic layers, where the differences of environmental factors are relatively small. 